\title{
Tinnitus distress is associated with enhanced resting-state functional connectivity within the default mode network
}

This article was published in the following Dove Press journal:

Neuropsychiatric Disease and Treatment

\author{
Yu-Chen Chen ${ }^{1,2}$ \\ Huiyou Chen ${ }^{2}$ \\ Fan $\mathrm{Bo}^{2}$ \\ Jin-Jing $X u^{3}$ \\ Yi Deng ${ }^{3}$ \\ Han Lv ${ }^{4}$ \\ Yuexin $\mathrm{Cai}^{5}$ \\ Wenqing $\mathrm{Xia}^{6}$ \\ Xindao Yin $^{2}$ \\ Jian-Ping $\mathrm{Gu}^{2}$ \\ Guangming Lu'
}

'Department of Medical Imaging, Jinling Hospital, School of Medicine, Nanjing University, Nanjing,

China; ${ }^{2}$ Department of Radiology,

Nanjing First Hospital, Nanjing

Medical University, Nanjing, China;

${ }^{3}$ Department of Otolaryngology,

Nanjing First Hospital, Nanjing

Medical University, Nanjing, China;

${ }^{4}$ Department of Radiology, Beijing

Friendship Hospital, Capital

Medical University, Beijing, China;

${ }^{5}$ Department of Otolaryngology, Sun

Yat-sen Memorial Hospital, Sun Yat-

sen University, Guangzhou, China;

${ }^{6}$ Department of Endocrinology,

Nanjing First Hospital, Nanjing

Medical University, Nanjing, China

Correspondence: Jian-Ping Gu Department of Radiology, Nanjing

First Hospital, Nanjing Medical

University, No 68, Changle Road,

Nanjing 210006 , China

Tel +862587726268

Fax +862552236361

Email cjr.gujianping@vip.163.com

Guangming Lu

Department of Medical Imaging, Jinling Hospital, School of Medicine, Nanjing University, No 305, Eastern Zhongshan

Road, Nanjing 210002, China

Email cjr.luguangming@vip.163.com
Purpose: The default mode network (DMN) has been confirmed to be involved in chronic tinnitus perception. Tinnitus distress may be associated with abnormal functional connectivity (FC) within the DMN regions. The goal of this study was to determine whether tinnitus disrupted the FC patterns within the DMN as measured by using resting-state functional magnetic resonance imaging approach.

Patients and methods: Resting-state functional magnetic resonance imaging scans were acquired from 40 chronic bilateral tinnitus patients and 41 healthy controls. Both were age, sex, and education well-matched with normal hearing. Two important DMN regions, the anterior cingulate cortex and posterior cingulate cortex, were chosen as seed regions to detect the FC patterns within the DMN and then determine whether these changes were linked to clinical measures of tinnitus such as tinnitus duration and tinnitus severity.

Results: Compared with healthy controls, chronic tinnitus patients manifested significantly enhanced FC between the anterior cingulate cortex and left precuneus, which was correlated with the tinnitus duration $(r=0.451, p=0.007)$. Moreover, enhanced FC between the posterior cingulate cortex and right medial prefrontal cortex in tinnitus patients was positively correlated with the tinnitus distress $(r=0.411, p=0.014)$.

Conclusion: Chronic tinnitus patients showed disrupted FC patterns within the DMN regions which are correlated with tinnitus distress. Increased resting-state connectivity pattern of the DMN may play a pivotal role in neuropathological features underlying chronic tinnitus.

Keywords: tinnitus, anterior cingulate cortex, posterior cingulate cortex, default mode network, resting-state fMRI

\section{Introduction}

Tinnitus is an auditory phantom percept of sound in the absence of an external source..$^{1,2}$ Of the 20 million people with tinnitus in USA, 2 million are extremely bothered and debilitated by the perception. ${ }^{3-5}$ Tinnitus patients often suffer from sleep disturbances, depression, anxiety, and other comorbid conditions that significantly impair the quality of daily life..$^{5-8}$ Prior studies have suggested that chronic tinnitus is associated with various neuropathological changes, such as enhanced spontaneous neuronal activity, aberrant consciousness gating, tonotopic map reorganization, increased neural synchrony, and coupling of networks involving auditory and non-auditory structures. ${ }^{9-14}$ Nevertheless, the neural mechanisms that maintain this disorder still remain poorly understood.

Resting-state functional magnetic resonance imaging (fMRI) has become a novel and widely used neuroimaging technique to explore the potential pathogenesis of various 
neuropsychiatric disorders. It is sensitive to low-frequency $(0.01-0.1 \mathrm{~Hz})$ spontaneous fluctuations in the blood oxygenation level-dependent (BOLD) signal with high spatial resolution and easy application, which represents baseline spontaneous neuronal activity. ${ }^{15-17}$ Moreover, resting-state networks (RSNs) are defined as brain regions with highly correlated time courses of low-frequency $(<0.1 \mathrm{~Hz})$ BOLD signal fluctuations during the baseline state. ${ }^{18}$ Prior studies have applied resting-state fMRI to examine multiple wholebrain RSNs, such as auditory network, attention network, and default mode network (DMN), to study the neural mechanisms of tinnitus. ${ }^{14,19,20}$ Among them, the DMN is most active at rest and shows reduced activity when a subject enters a task-based state involving attention or goal-directed behavior, which consists of nodes in the anterior cingulate cortex (ACC), posterior cingulate cortex (PCC)/precuneus, medial prefrontal cortex ( $\mathrm{mPFC}$ ), and inferior parietal lobule (IPL). ${ }^{21}$ Because of its persistent nature, some have proposed that the generation of tinnitus may be associated with the abnormalities in the DMN. ${ }^{19}$ Schmidt et al observed that the precuneus was found to have significantly decreased connectivity with other nodes of the DMN in tinnitus patients relative to control groups..$^{22,23}$ Other studies using independent component or graph theory analyses have demonstrated enhanced functional connectivity (FC) between the DMN and other brain networks in tinnitus patients. ${ }^{24,25}$ Enhanced FC was also observed between the DMN and other brain regions such as limbic system, which was associated with tinnitus distress. ${ }^{26}$ Moreover, other psychiatric disorders, such as posttraumatic stress disorder ${ }^{27}$ or Parkinson's disease, ${ }^{28}$ have also observed the associations between elevated connectivity within the DMN and disease-related distress. Thus, it is not surprising that this specific association is implicated in tinnitus as well.

Furthermore, as the core nodes of DMN, the ACC and PCC play important roles in emotional control and processing distressing information. ${ }^{29,30}$ During cognitive processing, the PCC is functionally related to ventral ACC and the DMNrelated brain region. ${ }^{30}$ Thus, the FC alterations of ACC and PCC might be associated with the cognitive deficit of tinnitus patients. Our previous studies have detected increased spontaneous neural activity in specific DMN regions of tinnitus including ACC and PCC. ${ }^{31-33}$ The DMN is relatively better connected internally (ie, larger spontaneous fluctuations, indicative of stronger connectivity) when the mind is at rest and allowed to wander or daydream. ${ }^{21}$ Nevertheless, none of the current studies have specifically revealed FC alterations within the DMN in chronic tinnitus. Disruptions of the DMN are not exclusive to tinnitus. Previous studies have noted FC disruptions primarily in anterior-to-posterior connections within the DMN in Alzheimer's disease. ${ }^{34,35}$ Moreover, results from studies of the DMN in major depressive disorder indicate a hyperactive network with increased FC changes that are associated with the process of rumination. ${ }^{36}$ However, the source of abnormal neural activity within specific DMN regions related to tinnitus still remains elusive.

Considering the crucial role of the DMN in the neuropathology of tinnitus, we aimed to employ a seed-based approach to investigate the intrinsic FC within the DMN regions between tinnitus patients with normal hearing and healthy controls. We hypothesized that resting-state FC patterns within the DMN regions in tinnitus would be significantly distinct from controls and that the enhanced FC within the DMN would correlate with specific tinnitus characteristics such as tinnitus distress and tinnitus duration.

\section{Patients and methods \\ Participants}

This study was approved by the Research Ethics Committee of the Nanjing Medical University. All individuals provided written informed consent before their participation in the study protocol.

A total of 40 bilateral tinnitus patients were recruited for this study at the Department of Otolaryngology of Nanjing First Hospital. Forty-one age-, sex-, and education-matched healthy controls were recruited through community health screening or newspaper advertisement. All participants were right-handed and completed at least 8 years of education. None of the participants was excluded because of exceeded limits for head motion during scanning. Tinnitus distress was assessed by the Iowa version of the Tinnitus Handicap Questionnaires (THQ). ${ }^{37}$ Based on the THQ score and previously proposed guidelines, ${ }^{38}$ the severity of the tinnitus patients was categorized as mild, moderate, or severe. Twenty patients had mild tinnitus, 17 had moderate tinnitus, and three had severe tinnitus in this study. Hearing thresholds were determined by pure tone audiometry (PTA) at the frequencies of $0.25,0.5,1,2,4$, and $8 \mathrm{kHz}$. None of the participants had hearing loss in any of the six measured audiometric frequencies (average PTA $\leq 25 \mathrm{~dB}$ ). There were no statistically significant differences in hearing thresholds between the two groups (Figure 1; Table 1). Depression and anxiety status were assessed according to the Self-Rating Depression Scale (SDS) and Self-Rating Anxiety Scale (SAS). ${ }^{39,40}$ According to a previous study, ${ }^{41}$ the Hyperacusis Questionnaire was used to exclude patients with hyperacusis 


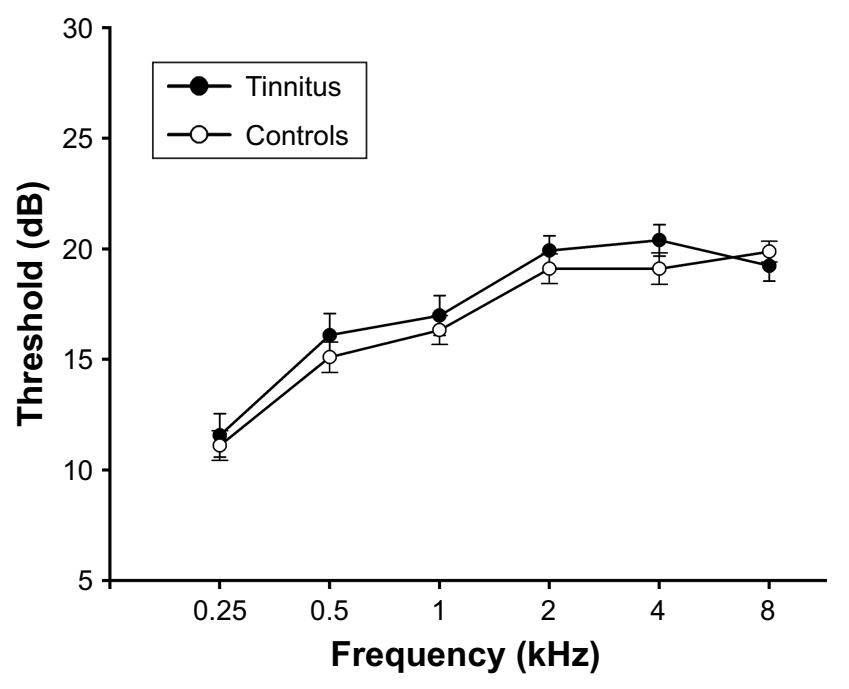

Figure I No significant differences in auditory thresholds between tinnitus and control groups.

Note: Data are presented as mean \pm SEM.

Abbreviation: SEM, standard error of the mean.

in this study. Participants were also excluded if they suffered from pulsatile tinnitus, hyperacusis, Meniere's diseases, or had a past history of otologic surgery, ototoxic drug therapy, noise exposure, or hearing aid use, severe smoking, stroke, alcoholism, brain injury, Parkinson's disease, Alzheimer's disease, epilepsy, major depression, neurological or psychiatric disorders that could affect cognitive function, major medical illness (eg, anemia, thyroid dysfunction, and cancer), MRI contraindications, or severe visual loss. The characteristics of the tinnitus patients and healthy controls are summarized in Table 1.

Table I Characteristics of tinnitus patients and healthy controls

\begin{tabular}{|c|c|c|c|}
\hline & $\begin{array}{l}\text { Tinnitus } \\
\text { patients } \\
(n=40)\end{array}$ & $\begin{array}{l}\text { Healthy } \\
\text { controls } \\
(n=4 I)\end{array}$ & $p$-value \\
\hline Age (years) & $53.6 \pm 12.5$ & $49.5 \pm 10.5$ & 0.110 \\
\hline Male/female & $19 / 21$ & $16 / 25$ & $0.44 I$ \\
\hline Education levels (years) & $12.5 \pm 2.4$ & $12.7 \pm 2.9$ & 0.670 \\
\hline Tinnitus duration (months) & $41.9 \pm 34.5$ & - & - \\
\hline THQ score & $52.1 \pm 15.9$ & - & - \\
\hline SDS score & $41.8 \pm 5.6$ & $40.0 \pm 4.6$ & 0.123 \\
\hline SAS score & $39.6 \pm 6.5$ & $37.0 \pm 5.6$ & 0.066 \\
\hline Gray matter & $578.8 \pm 25.6$ & $577.5 \pm 22.7$ & 0.815 \\
\hline White matter & $534.6 \pm 21.8$ & $526.7 \pm 25.6$ & 0.139 \\
\hline Brain parenchyma & $1,113.4 \pm 32.1$ & $\mathrm{I}, 104.2 \pm 35.4$ & 0.225 \\
\hline Hearing thresholds (left) & $15.8 \pm 3.5$ & $16.7 \pm 2.5$ & 0.093 \\
\hline Hearing thresholds (right) & $166 \pm 3.4$ & $17.0 \pm 2.2$ & 0.592 \\
\hline Hearing thresholds (average) & $16.1 \pm 3.0$ & $16.8 \pm 1.5$ & 0.158 \\
\hline FD value $(\mathrm{mm})$ & $0.21 \pm 0.06$ & $0.21 \pm 0.07$ & 0.952 \\
\hline
\end{tabular}

Note: Data are represented as mean \pm SD.

Abbreviations: THQ, Tinnitus Handicap Questionnaire; SDS, Self-Rating Depression Scale; SAS, Self-Rating Anxiety Scale; FD, framewise displacement.

\section{MR acquisition}

MRI data were acquired by using a 3.0 T MRI scanner (Ingenia; Philips Medical Systems, the Netherlands) with an eight-channel receiver array head coil. Head motion and scanner noise were alleviated by using foam padding and earplugs. The earplugs (Hearos Ultimate Softness Series, Aliso Viejo, CA, USA) were used to attenuate scanner noise by $\sim 32 \mathrm{~dB}$ based on the manufacture's data sheet. The participants were instructed to lie down quietly with their eyes closed and without falling asleep, to not think about anything in particular, and to avoid any head motion during the scan. Functional images were obtained axially by using a gradient echo-planar imaging sequence as follows: repetition time $(\mathrm{TR})=2,000 \mathrm{~ms}$; echo time $(\mathrm{TE})=30 \mathrm{~ms}$; slices $=36$; thickness $=4 \mathrm{~mm}$; gap $=0 \mathrm{~mm}$; field of view $(F O V)=240 \times 240 \mathrm{~mm}$; acquisition matrix $=64 \times 64$; and flip angle $(\mathrm{FA})=90^{\circ}$. The fMRI sequence took 8 minutes and 8 seconds. All scans were acquired with parallel imaging by using sensitivity encoding (SENSE) technique, SENSE factor $=2$. Structural images were acquired with a three-dimensional turbo fast echo T1WI sequence with high resolution as follows: $\mathrm{TR} / \mathrm{TE}=8.1 / 3.7 \mathrm{~ms}$; slices $=170$; thickness $=1 \mathrm{~mm}$; gap $=0 \mathrm{~mm} ; \mathrm{FA}=8^{\circ}$; acquisition matrix $=256 \times 256 ; \mathrm{FOV}=256 \times 256 \mathrm{~mm}^{2}$. The structural sequence took 5 minutes and 29 seconds.

\section{Data preprocessing}

fMRI data preprocessing was carried out by using the software tool Data Processing Assistant for Resting-State fMRI programs ${ }^{42}$ which is based on Statistical Parametric Mapping (SPM12, http://www.fil.ion.ucl.ac.uk/spm) and the resting-state fMRI data analysis toolkit (REST, http://www. restfmri.net). The first 10 volumes were removed from each time series to account for the time it took participants to adapt to the scanning environment. Slice timing and realignment for head-motion correction were then performed for the remaining 230 images. Participant data exhibiting head motion $>2.0 \mathrm{~mm}$ translation or $>2.0^{\circ}$ rotation were excluded from the analysis. The remaining data set was spatially normalized to the Montreal Neurological Institute template (resampling voxel size $=3 \times 3 \times 3 \mathrm{~mm}^{3}$ ), smoothing with an isotropic Gaussian kernel (full width at half maximum $[\mathrm{FWHM}]=6 \mathrm{~mm})$, detrending and filtering $(0.01-0.08 \mathrm{~Hz})$ were performed in order.

\section{Structural data analysis}

Structural images of each subject were processed by using the VBM toolbox software in SPM12 (http://www.fil. ion.ucl.ac.uk/spm). Briefly, the structural images were 
normalized and segmented into gray matter (GM), white matter (WM), and cerebrospinal fluid (CSF) by using the unified segmentation model in SPM12. ${ }^{43}$ Brain parenchyma volume was calculated as the sum of GM and WM volumes. T1 images were normalized to the MNI template by using affine linear registration followed by Gaussian smoothing (FWHM =8 mm).

\section{Functional data analysis}

The seed regions of interest (ROIs) of the ACC and PCC were generated from Brodmann template by using the WFU_PickAtlas software. ${ }^{44}$ Briefly, the mean time series of each ROI was obtained for reference time course. Then, the Pearson's correlation coefficients were calculated between the mean signal change of each ROI and the time series of each voxel. Finally, a Fisher's $z$-transform was used to improve the normality of the correlation coefficients. ${ }^{45}$ Six head motion parameters and mean time courses of global, WM, and CSF signals were included in the regression analysis.

One-sample $t$-test was performed on the individual FC maps in a voxel-wise manner. Each individual's $z$ map was entered into the SPM12 software for a random effect one-sample $t$-test to determine the brain regions showing significant FC to ACC and PCC. Significant thresholds were corrected by using false discovery rate (FDR) criterion and set at $p<0.001$, based on the suggestion from a prior study. ${ }^{46}$ Two-sample $t$-tests were performed to investigate FC differences between tinnitus patients and controls by using a default whole-brain mask. Age, sex, education, GM volume, and mean hearing thresholds were used as nuisance covariates. The voxel-wise results of group difference were corrected for multiple comparisons by using FDR criterion and the significance set at $p<0.001$.

\section{Statistical analysis}

Between-group $t$-tests and $\chi^{2}$ tests were used to analyze the differences in the demographic data between tinnitus patients and healthy controls. To identify the relationship between abnormal FC and clinical variables, Pearson correlation analyses were performed in a ROI-wise manner. The mean $z$-values of each brain region that showed significant group differences were extracted within every subject. Pearson correlation analysis between the mean $z$-values and each clinical variable was performed by using SPSS 19.0 (SPSS Inc., Chicago, IL, USA). Statistical threshold was set at $p<0.05$. Partial correlations between extracted values and clinical variables were calculated using the SPSS software. Analyses were adjusted for age, sex, education, GM volume, mean hearing thresholds, and specific clinical variables. Bonferroni correction was applied for multiple comparisons in the correlation analyses. The Bonferroni corrected $p$-value was 0.0125 .

Since micromovements from volume to volume can influence the $\mathrm{FC},{ }^{47}$ framewise displacement (FD) values were computed for each subject to reflect the temporal derivative of the movement parameters. No subjects had FD $>0.5 \mathrm{~mm}$ on more than 35 volumes in this study. No significant difference was found in the mean FD values between tinnitus patients and controls (Table 1).

\section{Results}

\section{Structural results}

Table 1 shows no significant differences in GM and WM volumes in tinnitus patients compared with healthy controls $(p>0.05)$. After Monte Carlo simulation correction, no suprathreshold voxel-wise difference in the GM and WM volume between tinnitus patients and healthy controls was observed.

\section{Functional analysis}

The ACC and PCC mainly showed positive FC within the DMN regions, including the $\mathrm{mPFC}$, IPL, and precuneus in both the tinnitus patients (Figure 2A) and healthy controls (Figure 2B). Relative to healthy controls, bilateral tinnitus patients exhibited significantly enhanced FC between the ACC and left precuneus as well as increased FC between PCC and right mPFC (Figure 3; Table 2).

\section{Correlation analysis results}

In bilateral tinnitus patients, the enhanced FC of ACC-left precuneus was positively correlated with the tinnitus duration ( $r=0.455, p=0.007$ ) (Figure 4A; Table 3 ), which could persist after Bonferroni correction. Furthermore, the increased FC of the PCC to right mPFC was positively associated with the THQ score ( $r=0.381, p=0.026$ ) (Figure 4B; Table 3), which could not persist after Bonferroni correction, probably partly due to the relatively strict calculation. These correlations had been corrected for age, sex, education, GM volume, and mean hearing thresholds. None of the increased FC was correlated with SAS or SDS score (Table 3).

\section{Discussion}

In the current study, we used the seed-based method to investigate disrupted DMN patterns in patients with bilateral chronic tinnitus. Compared with healthy subjects, chronic tinnitus patients revealed significantly enhanced $\mathrm{FC}$ between 

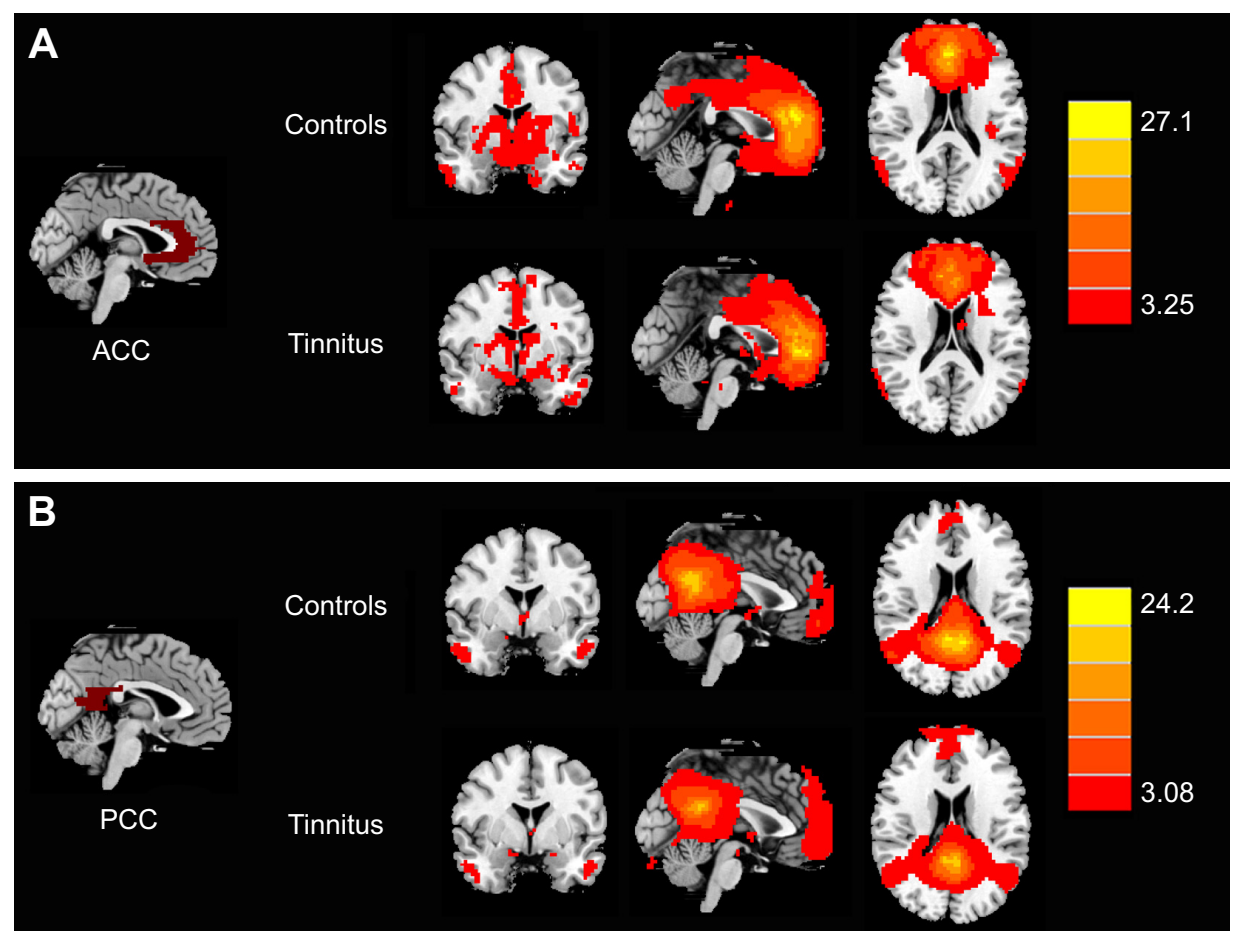

Figure 2 Significant FC patterns from the ROls of the (A) ACC and (B) PCC in whole brain by using one-sample t-test in both chronic tinnitus patients and healthy controls. Note: Significant thresholds were corrected by using FDR criterion and set at $p<0.00 \mathrm{I}$.

Abbreviations: FC, functional connectivity; ROI, region of interest; ACC, anterior cingulate cortex; PCC, posterior cingulate cortex; FDR, false discovery rate.

the ACC and left precuneus, which was correlated with the tinnitus duration. Moreover, enhanced FC between the PCC and right $\mathrm{mPFC}$ in tinnitus patients was positively correlated with the tinnitus distress.

In this study, we compared GM and WM volumes but did not detect any brain structural changes between the normal hearing tinnitus patients and matched healthy controls, which was consistent with our prior studies. ${ }^{26,31-33,48,49}$ By using VBM or surface-based morphometry, prior studies have observed decreases of GM volume in tinnitus patients

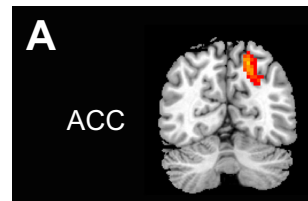
$x=-17$

B

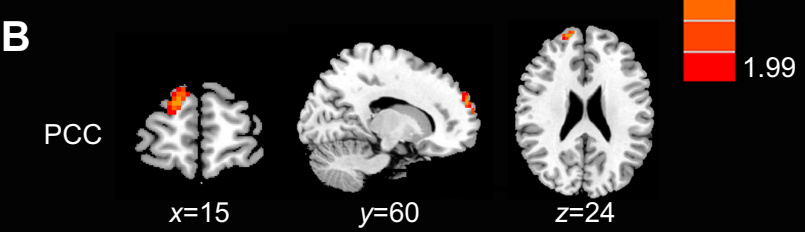

Figure 3 Aberrant FC of the (A) ACC and (B) PCC in tinnitus patients compared with healthy controls.

Note: The threshold was set at a $p<0.00$ I (FDR corrected).

Abbreviations: FC, functional connectivity; ACC, anterior cingulate cortex; PCC, posterior cingulate cortex; FDR, false discovery rate. in several brain regions including $\mathrm{ACC}, \mathrm{mPFC}$, inferior colliculus, hippocampus, superior frontal gyrus, occipital lobe, and hypothalamus. ${ }^{50-58}$ Increases in GM volume have also been reported in tinnitus patients in the superior and middle temporal gyrus. ${ }^{57}$ The possible explanation for this is the absence of hearing loss over the extended frequency range and absence of hyperacusis in our tinnitus patients. Moreover, the MR analytical technique could also contribute to the discrepancy.

Patients with chronic tinnitus had significantly greater FC between the ACC and left precuneus, which was correlated with tinnitus duration. We suggest that the ACC is part of the core structure of anterior DMN, which may show functional dysconnectivity with the precuneus in tinnitus. Precuneus

Table 2 Abnormal functional connectivity of ACC and PCC in bilateral tinnitus patients compared with healthy controls

\begin{tabular}{lcccc}
\hline Brain region & BA & $\begin{array}{l}\text { MNI coordinates } \\
\mathbf{x}, \mathbf{y}, \mathbf{z}(\mathbf{m m})\end{array}$ & T score & Voxels \\
\hline ACC & 7 & $-17,-64,46$ & 3.4215 & 80 \\
$\begin{array}{l}\text { L precuneus } \\
\text { PCC }\end{array}$ & & & 3.4359 & 62 \\
\hline R medial prefrontal cortex & 10 & $15,60,24$ & 6 \\
\hline
\end{tabular}

Note: The threshold was set at a $p<0.001$ (FDR corrected).

Abbreviations: ACC, anterior cingulate cortex; PCC, posterior cingulate cortex; BA, Brodmann's area; MNI, Montreal Neurological Institute; L, left; R, right; FDR, false discovery rate. 

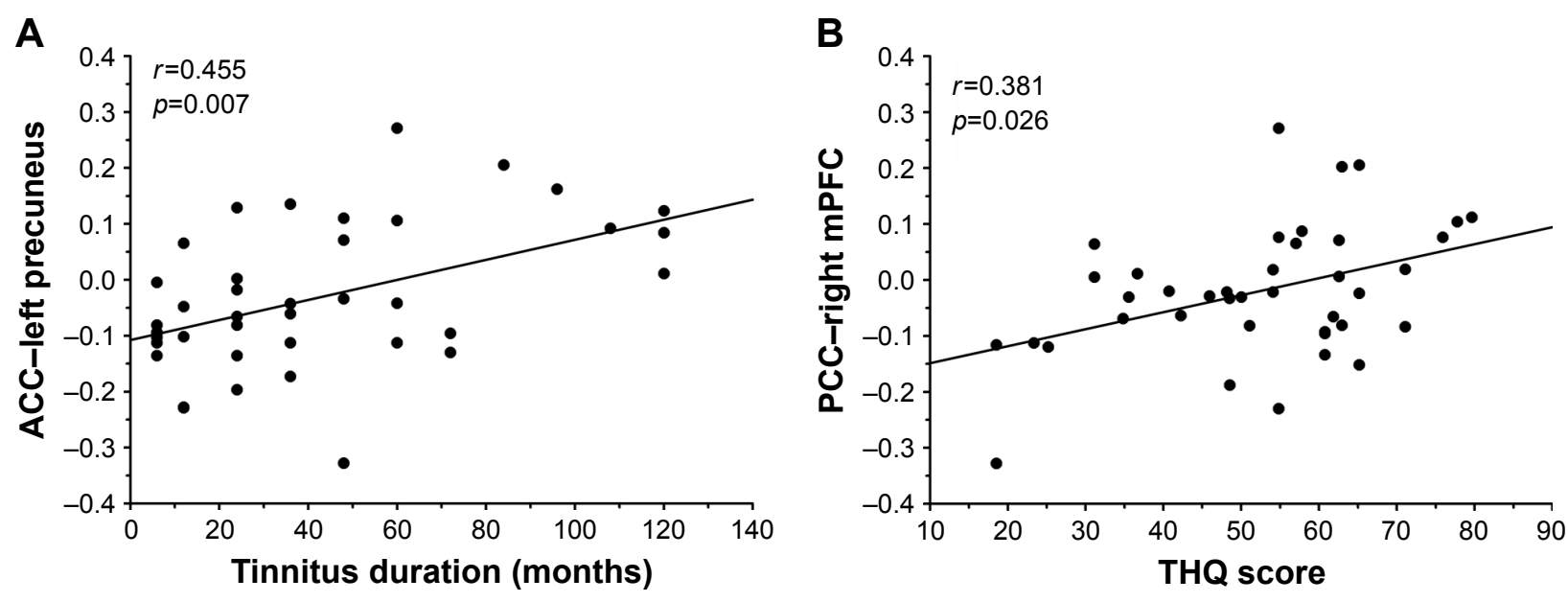

Figure 4 Significant correlations between the enhanced FC and tinnitus characteristics.

Note: (A) Positive correlation between the increased FC of the ACC-left precuneus and the tinnitus duration. (B) Positive correlation between the increased FC of the PCC-right mPFC and the THQ score.

Abbreviations: FC, functional connectivity; ACC, anterior cingulate cortex; PCC, posterior cingulate cortex; mPFC, medial prefrontal cortex; THQ, Tinnitus Handicap Questionnaires.

is a highly integrated structure, supposed to be involved in self-consciousness, shifting of attention, auditory memory retrieval, auditory imagery, and memory-related aspects of the tinnitus perception. ${ }^{59,60}$ Moreover, precuneus is one of the important regions in brain that are linked with tinnitus distress. ${ }^{19,61-63}$ However, in this study, the patients maintained higher connectivity with the left precuneus that was correlated with tinnitus duration, indicating preservation of normal episodic memory, despite the presence of persistent tinnitus percept. ${ }^{64,65}$ Furthermore, some research studies show that a positive correlation between the strength of inflow to the temporal cortex and tinnitus distress was found in DMN areas, especially in the precuneus. ${ }^{66}$ Despite the correlation with the tinnitus duration or tinnitus distress, these results all imply that the aberrant activity of precuneus within the DMN may be a potential marker of chronic tinnitus. Our current evidence suggests that the elevated connectivity within the $\mathrm{DMN}$ is a long-term effect and therefore a consequence (not a cause) from chronic tinnitus. Previous studies have also demonstrated aberrant FC changes exclusively within the DMN in patients with long-term tinnitus compared with healthy controls, ${ }^{22,23,67}$ suggesting that this disruption within the DMN is not immediate but occurs over time in tinnitus patients. In addition, the existing literature seems to indicate that tinnitus severity has a significant impact on the brain resting-state FC networks within the DMN. ${ }^{19,24,25,68}$ Besides that, the precuneus has also been suggested to be related to conscious and internal awareness and may play a key role in tinnitus generation and persistence. ${ }^{69,70}$ Therefore, consistent with the aforementioned studies, our results suggest that abnormal FC between the ACC and precuneus may be responsible for disrupting the DMN in chronic tinnitus patients. Nevertheless, the source of abnormal neural activity within specific DMN regions due to tinnitus still remains unknown.

Alongside impaired connectivity in the precuneus, patients with chronic tinnitus also showed increased connectivity between the PCC and MPFC. This finding is similar to prior fMRI studies that have reported enhanced connectivity in the mPFC among tinnitus patients. ${ }^{14,26,33,54,71-73}$ The $\mathrm{mPFC}$ has been regarded as a pivotal region that integrates sensory and emotional aspects of tinnitus. ${ }^{74}$ Rauschecker et al developed a model to identify structural and functional differences in ventromedial prefrontal cortex which were related to tinnitus loudness, indicating the contribution of $\mathrm{mPFC}$ to certain perceptual features of tinnitus. ${ }^{71}$ Moreover, Araneda

Table 3 Correlation coefficients between the increased functional connectivity of ACC and PCC and different tinnitus characteristics

\begin{tabular}{lllll}
\hline Brain region & Tinnitus duration & THQ & SDS & SAS \\
\hline ACC-left precuneus & $r=0.455, p=0.007^{*}$ & $r=0.078, p=0.661$ & $r=-0.082, p=0.690$ & $r=-0.336, p=0.094$ \\
PCC-right mPFC & $r=-0.115, p=0.518$ & $r=0.381, p=0.026^{*}$ & $r=-0.028, p=0.891$ & $r=0.296, p=0.142$ \\
\hline
\end{tabular}

Notes: Partial correlations were controlled for age, sex, education, GM volume, and mean hearing thresholds. ${ }^{*} p<0.05$.

Abbreviations: ACC, anterior cingulate cortex; PCC, posterior cingulate cortex; mPFC, medial prefrontal cortex; THQ, Tinnitus Handicap Questionnaires; SDS, Self-Rating Depression Scale; SAS, Self-Rating Anxiety Scale. 
et al indicated that deficit in executive functions caused by $\mathrm{mPFC}$ alterations might be a key factor in the generation and maintenance of tinnitus. ${ }^{72}$ Ahn et al suggested that tinnitus might be related to impaired prefrontal top-down inhibitory control using resting-state electroencephalography. ${ }^{75}$ Moreover, increased FC strength between auditory and $\mathrm{PFC}$ regions was observed through resting-state functional near-infrared spectroscopy. ${ }^{76}$ In addition, our previous study also observed significantly enhanced FC within the executive control of attention network involving the mPFC region. ${ }^{32}$ However, in other studies with larger sample size, chronic tinnitus-related distress was not correlated with morphometric or functional changes in the PFC..$^{50,51,58,77}$ The heterogeneity of the tinnitus population and the different MR techniques may contribute to the discrepancies. Thus, our findings of increased FC between the posterior DMN region and $\mathrm{mPFC}$ might suggest that the $\mathrm{MPFC}$ serves as a main cortical hub in the brain network influenced by chronic tinnitus.

There were some limitations in the current study. First, this is a cross-sectional study with a limited sample size. Further longitudinal investigations are needed to confirm the relationships between aberrant FC patterns within the DMN and developing tinnitus distress in future study. Second, the scanner noise cannot be completely eliminated even with earplugs, which may reduce the differences in RSNs between tinnitus patients and controls. This confounding factor should be taken into consideration in future studies. Moreover, besides the functional dysconnectivity, more research studies are needed to demonstrate the possibility of structural connectivity within the DMN that can be detected by using the diffusion tensor imaging approach. Besides the VBM analysis, surface-based methods such as FreeSurfer or related methods may be more precise and informative for providing cortical thickness, surface area, and volume, which need to be considered in our future study. Finally, subtypes of different age, severity, and other confounding factors that could result in a deviation of tinnitus require to be divided to prevent the disordered effect of the inconformity of these subgroups.

\section{Conclusion}

Despite these limitations, this study demonstrated enhanced resting-state FC patterns within the DMN, which was associated with the tinnitus-related distress. These findings mainly illustrated the potential role of DMN in chronic tinnitus patients which will contribute to a better understanding of the neuropathological mechanisms in tinnitus perception.

\section{Acknowledgments}

This work was supported by a grant from the National Natural Science Foundation of China (no 81601477), Natural Science Foundation of Jiangsu Higher Education Institutions (no 16KJB320001), Medical Science and Technology Development Foundation of Nanjing Department of Health (no YKK16140), Youth Medical Talents of Jiangsu Province (no QNRC2016062), China Postdoctoral Science Foundation (no 2017M610337), Jiangsu Postdoctoral Science Foundation (no 1701007A), 14th "Six Talent Peaks" Project of Jiangsu Province (no YY-079), and Nanjing Outstanding Youth Fund (no JQX17006).

\section{Disclosure}

The authors report no conflicts of interest in this work.

\section{References}

1. Wegger M, Ovesen T, Larsen DG. Acoustic coordinated reset neuromodulation: a systematic review of a novel therapy for tinnitus. Front Neurol. 2017;8:36.

2. Møller AR. Tinnitus: presence and future. Prog Brain Res. 2007; 166:3-16.

3. Henry JA, Dennis KC, Schechter MA. General review of tinnitus: prevalence, mechanisms, effects, and management. J Speech Lang Hear Res. 2005;48:1204-1235.

4. Hall DA, Lainez MJ, Newman CW, et al. Treatment options for subjective tinnitus: self reports from a sample of general practitioners and ENT physicians within Europe and the USA. BMC Health Serv Res. 2011;11:302.

5. Langguth B, Kreuzer PM, Kleinjung T, De Ridder D. Tinnitus: causes and clinical management. Lancet Neurol. 2013;12:920-930.

6. Reynolds P, Gardner D, Lee R. Tinnitus and psychological morbidity: a cross-sectional study to investigate psychological morbidity in tinnitus patients and its relationship with severity of symptoms and illness perceptions. Clin Otolaryng Allied Sci. 2004;29:628-634.

7. Zeman F, Koller M, Langguth B, Landgrebe M. Which tinnitus-related aspects are relevant for quality of life and depression: results from a large international multicentre sample. Health Qual Life Outcomes. 2014;12:7.

8. Langguth B. A review of tinnitus symptoms beyond "ringing in the ears": a call to action. Curr Med Res Opin. 2011;27:1635-1643.

9. Kaltenbach JA, Zhang J, Finlayson P. Tinnitus as a plastic phenomenon and its possible neural underpinnings in the dorsal cochlear nucleus. Hear Res. 2005;206:200-226.

10. Lockwood AH, Salvi RJ, Coad ML, Towsley ML, Wack DS, Murphy BW. The functional neuroanatomy of tinnitus: evidence for limbic system links and neural plasticity. Neurology. 1998;50:114-120.

11. Norena A, Eggermont J. Changes in spontaneous neural activity immediately after an acoustic trauma: implications for neural correlates of tinnitus. Hear Res. 2003;183:137-153.

12. Noreña AJ, Farley BJ. Tinnitus-related neural activity: theories of generation, propagation, and centralization. Hear Res. 2013;295:161-171.

13. Zeng F-G. An active loudness model suggesting tinnitus as increased central noise and hyperacusis as increased nonlinear gain. Hear Res. 2013;295:172-179.

14. Chen Y-C, Wang F, Wang J, et al. Resting-state brain abnormalities in chronic subjective tinnitus: a meta-analysis. Front Hum Neurosci. 2017;11:22.

15. Fox MD, Raichle ME. Spontaneous fluctuations in brain activity observed with functional magnetic resonance imaging. Nat Rev Neurosci. 2007; 8:700-711. 
16. Biswal B, Yetkin FZ, Haughton VM, Hyde JS. Functional connectivity in the motor cortex of resting human brain using echo-planar MRI. Magn Reson Med. 1995;34:537-541.

17. Ogawa S, Lee TM, Kay AR, Tank DW. Brain magnetic resonance imaging with contrast dependent on blood oxygenation. Proc Natl Acad Sci US A. 1990;87:9868-9872.

18. Rosazza C, Minati L. Resting-state brain networks: literature review and clinical applications. Neurol Sci. 2011;32:773-785.

19. Husain FT, Schmidt SA. Using resting state functional connectivity to unravel networks of tinnitus. Hear Res. 2014;307:153-162.

20. Leaver AM, Turesky TK, Seydell-Greenwald A, Morgan S, Kim HJ, Rauschecker JP. Intrinsic network activity in tinnitus investigated using functional MRI. Hum Brain Mapp. 2016;37:2717-2735.

21. Raichle ME, MacLeod AM, Snyder AZ, Powers WJ, Gusnard DA, Shulman GL. A default mode of brain function. Proc Natl Acad Sci US A. 2001;98:676-682.

22. Schmidt SA, Carpenter-Thompson J, Husain FT. Connectivity of precuneus to the default mode and dorsal attention networks: a possible invariant marker of long-term tinnitus. Neuroimage Clin. 2017;16:196-204.

23. Schmidt SA, Akrofi K, Carpenter-Thompson JR, Husain FT. Default mode, dorsal attention and auditory resting state networks exhibit differential functional connectivity in tinnitus and hearing loss. PLoS One. 2013;8:e76488.

24. Burton H, Wineland A, Bhattacharya M, Nicklaus J, Garcia KS, Piccirillo JF. Altered networks in bothersome tinnitus: a functional connectivity study. BMC Neurosci. 2012;13:3.

25. Maudoux A, Lefebvre P, Cabay J-E, et al. Connectivity graph analysis of the auditory resting state network in tinnitus. Brain Res. 2012;1485:10-21.

26. Chen YC, Xia W, Chen H, et al. Tinnitus distress is linked to enhanced resting-state functional connectivity from the limbic system to the auditory cortex. Hum Brain Mapp. 2017;38:2384-2397.

27. King AP, Block SR, Sripada RK, et al. Altered default mode network $(\mathrm{DMN})$ resting state functional connectivity following a mindfulnessbased exposure therapy for posttraumatic stress disorder (PTSD) in combat veterans of Afghanistan and Iraq. Depress Anxiety. 2016;33:289-299.

28. Tessitore A, Giordano A, De Micco R, et al. Functional connectivity underpinnings of fatigue in "Drug-Naïve" patients with Parkinson's disease. Mov Disord. 2016;31:1497-1505.

29. Bush G, Luu P, Posner MI. Cognitive and emotional influences in anterior cingulate cortex. Trends Cogn Sci. 2000;4:215-222.

30. Fransson P, Marrelec G. The precuneus/posterior cingulate cortex plays a pivotal role in the default mode network: evidence from a partial correlation network analysis. Neuroimage. 2008;42:1178-1184.

31. Chen Y-C, Zhang J, Li X-W, et al. Aberrant spontaneous brain activity in chronic tinnitus patients revealed by resting-state functional MRI. Neuroimage Clin. 2014;6:222-228.

32. Chen Y-C, Zhang J, Li X-W, et al. Altered intra- and interregional synchronization in resting-state cerebral networks associated with chronic tinnitus. Neural Plast. 2015;2015:475382.

33. Chen Y-C, Liu S, Lv H, et al. Abnormal resting-state functional connectivity of the anterior cingulate cortex in unilateral chronic tinnitus patients. Front Neurosci. 2018;12:9.

34. Sala-Llonch R, Bartres-Faz D, Junque C. Reorganization of brain networks in aging: a review of functional connectivity studies. Front Psychol. 2015;6:663.

35. Buckner RL, Andrews-Hanna JR, Schacter DL. The brain's default network: anatomy, function, and relevance to disease. Ann N Y Acad Sci. 2008;1124:1-38.

36. Zhang K, Zhu Y, Wu S, et al. Molecular, functional, and structural imaging of major depressive disorder. Neurosci Bull. 2016;32:273-285.

37. Kuk FK, Tyler RS, Russell D, Jordan H. The psychometric properties of a Tinnitus Handicap Questionnaire. Ear Hear. 1990;11:434-445.

38. McCombe A, Baguley D, Coles R, McKenna L, McKinney C, WindleTaylor P. Guidelines for the grading of tinnitus severity: the results of a working group commissioned by the British Association of Otolaryngologists, Head and Neck Surgeons, 1999. Clin Otolaryngol. 2001; 26(5):388-393.
39. Zung W. Zung self-rating depression scale and depression status inventory. In: Sartorius N, Ban TA, editors. Assessment of Depression. Berlin, Heidelberg: Springer; 1986:221-231.

40. Zung WW. A rating instrument for anxiety disorders. Psychosomatics. 1971;12:371-379.

41. Khalfa S, Dubal S, Veuillet E, Perez-Diaz F, Jouvent R, Collet L. Psychometric normalization of a hyperacusis questionnaire. ORL J Otorhinolaryngol Relat Spec. 2002;64:436-442.

42. Chao-Gan Y, Yu-Feng Z. DPARSF: a MATLAB toolbox for "pipeline" data analysis of resting-state fMRI. Front Syst Neurosci. 2010; $4: 13$.

43. Ashburner J, Friston KJ. Unified segmentation. Neuroimage. 2005;26: 839-851.

44. Maldjian JA, Laurienti PJ, Kraft RA, Burdette JH. An automated method for neuroanatomic and cytoarchitectonic atlas-based interrogation of fMRI data sets. Neuroimage. 2003;19:1233-1239.

45. Lowe MJ, Mock BJ, Sorenson JA. Functional connectivity in single and multislice echoplanar imaging using resting-state fluctuations. Neuroimage. 1998;7:119-132.

46. Eklund A, Nichols TE, Knutsson H. Cluster failure: why fMRI inferences for spatial extent have inflated false-positive rates. Proc Natl Acad Sci U S A. 2016;113:7900-7905.

47. Power JD, Barnes KA, Snyder AZ, Schlaggar BL, Petersen SE. Spurious but systematic correlations in functional connectivity MRI networks arise from subject motion. Neuroimage. 2012;59:2142-2154.

48. Chen YC, Xia W, Feng Y, et al. Altered interhemispheric functional coordination in chronic tinnitus patients. BioMed Res Int. 2015;2015: 345647.

49. Feng Y, Chen YC, Lv H, et al. Increased resting-state cerebellar-cerebral functional connectivity underlying chronic tinnitus. Front Aging Neurosci. 2018;10:59.

50. Allan TW, Besle J, Langers DR, et al. Neuroanatomical alterations in tinnitus assessed with magnetic resonance imaging. Front Aging Neurosci. 2016;8:221

51. Yoo HB, De Ridder D, Vanneste S. The importance of aging in gray matter changes within tinnitus patients shown in cortical thickness, surface area and volume. Brain Topogr. 2016;29:885-896.

52. Aldhafeeri FM, Mackenzie I, Kay T, Alghamdi J, Sluming V. Neuroanatomical correlates of tinnitus revealed by cortical thickness analysis and diffusion tensor imaging. Neuroradiology. 2012;54:883-892.

53. Leaver AM, Seydell-Greenwald A, Turesky TK, Morgan S, Kim HJ, Rauschecker JP. Cortico-limbic morphology separates tinnitus from tinnitus distress. Front Syst Neurosci. 2012;6:21.

54. Seydell-Greenwald A, Leaver AM, Turesky TK, Morgan S, Kim HJ, Rauschecker JP. Functional MRI evidence for a role of ventral prefrontal cortex in tinnitus. Brain Res. 2012;1485:22-39.

55. Muhlau M, Rauschecker JP, Oestreicher E, et al. Structural brain changes in tinnitus. Cereb Cortex. 2006;16:1283-1288.

56. Landgrebe M, Langguth B, Rosengarth K, et al. Structural brain changes in tinnitus: grey matter decrease in auditory and non-auditory brain areas. Neuroimage. 2009;46:213-218.

57. Boyen K, Langers DR, de Kleine E, van Dijk P. Gray matter in the brain: differences associated with tinnitus and hearing loss. Hear Res. 2013; 295:67-78.

58. Meyer M, Neff P, Liem F, et al. Differential tinnitus-related neuroplastic alterations of cortical thickness and surface area. Hear Res. 2016;342:1-12.

59. Yoo S-S, Lee CU, Choi BG. Human brain mapping of auditory imagery: event-related functional MRI study. Neuroreport. 2001;12:3045-3049.

60. Buckner RL, Raichle ME, Miezin FM, Petersen SE. Functional anatomic studies of memory retrieval for auditory words and visual pictures. J Neurosci. 1996;16(19):6219-6235.

61. Husain FT. Neural networks of tinnitus in humans: elucidating severity and habituation. Hear Res. 2016;334:37-48.

62. Pattyn T, Van Den Eede F, Vanneste S, et al. Tinnitus and anxiety disorders: a review. Hear Res. 2016;333:255-265. 
63. Han L, Zhaohui L, Fei Y, et al. Abnormal baseline brain activity in patients with pulsatile tinnitus: a resting-state FMRI study. Neural Plast. 2014;2014:549162.

64. Lundstrom BN, Petersson KM, Andersson J, Johansson M, Fransson P, Ingvar M. Isolating the retrieval of imagined pictures during episodic memory: activation of the left precuneus and left prefrontal cortex. Neuroimage. 2003;20:1934-1943.

65. Han L, Zhaohui L, Fei Y, et al. Disrupted neural activity in unilateral vascular pulsatile tinnitus patients in the early stage of disease: evidence from resting-state fMRI. Progress in Neuro-Psychopharmacology and Biological Psychiatry. 2015;59:91-99.

66. Schlee W, Mueller N, Hartmann T, Keil J, Lorenz I, Weisz N. Mapping cortical hubs in tinnitus. BMC Biol. 2009;7:80.

67. Carpenter-Thompson JR, Schmidt SA, Husain FT. Neural plasticity of mild tinnitus: an fMRI investigation comparing those recently diagnosed with tinnitus to those that had tinnitus for a long period of time. Neural Plast. 2015;2015:161478.

68. Wineland AM, Burton H, Piccirillo J. Functional connectivity networks in nonbothersome tinnitus. Otolaryngol Head Neck Surg. 2012;147: 900-906.

69. Cavanna AE, Trimble MR. The precuneus: a review of its functional anatomy and behavioural correlates. Brain. 2006;129:564-583.
70. Cavanna AE. The precuneus and consciousness. CNS Spectr. 2007;12: 545-552.

71. Rauschecker JP, Leaver AM, Muhlau M. Tuning out the noise: limbicauditory interactions in tinnitus. Neuron. 2010;66:819-826.

72. Araneda R, Renier L, Dricot L, et al. A key role of the prefrontal cortex in the maintenance of chronic tinnitus: an fMRI study using a Stroop task. Neuroimage Clin. 2018;17:325-334.

73. Chen Y-C, Feng Y, Xu J-J, et al. Disrupted brain functional network architecture in chronic tinnitus patients. Front Aging Neurosci. 2016; $8: 174$.

74. Jastreboff PJ. Phantom auditory perception (tinnitus): mechanisms of generation and perception. Neurosci Res. 1990;8:221-254.

75. Ahn M-H, Hong SK, Min B-K. The absence of resting-state high-gamma cross-frequency coupling in patients with tinnitus. Hear Res. 2017; 356:63-73.

76. San Juan J, Hu X-S, Issa M, et al. Tinnitus alters resting state functional connectivity (RSFC) in human auditory and non-auditory brain regions as measured by functional near-infrared spectroscopy (fNIRS). PLoS One. 2017;12:e0179150.

77. Schecklmann M, Landgrebe M, Poeppl TB, et al. Neural correlates of tinnitus duration and distress: a positron emission tomography study. Hum Brain Mapp. 2013;34:233-240.
Neuropsychiatric Disease and Treatment

\section{Publish your work in this journal}

Neuropsychiatric Disease and Treatment is an international, peerreviewed journal of clinical therapeutics and pharmacology focusing on concise rapid reporting of clinical or pre-clinical studies on a range of neuropsychiatric and neurological disorders. This journal is indexed on PubMed Central, the 'PsycINFO' database and CAS,

\section{Dovepress}

and is the official journal of The International Neuropsychiatric Association (INA). The manuscript management system is completely online and includes a very quick and fair peer-review system, which is all easy to use. Visit http://www.dovepress.com/testimonials.php to read real quotes from published authors. 